\title{
A Service Mashup Tool for Open Document Collaboration
}

\author{
Nelly Schuster, Raffael Stein, Christian Zirpins, and Stefan Tai \\ eOrganization Group, Karlsruhe Institute of Technology (KIT), \\ Englerstraße 11, Karlsruhe, Germany \\ \{firstname. lastname\} akit.edu
}

\begin{abstract}
Mashup technologies enable end-users to compose situational applications from Web-based services. A particular problem is to find high-level service composition models that a) are intuitive and expressive enough to be easily used by end-users and b) allow for efficient runtime support and integration on the Web. We propose a novel approach that leverages a metaphor of document collaboration: end-users declare the coordination and aggregation of peer contributions into a joint document. Peers provide contributions as Webbased services that allow a) integrating any Web-accessible resource and b) orchestrating the collaboration process. Thus, collaborative document mashups enable lightweight, situational collaboration that is not addressed by most BPM or CSCW systems. In this demo we present our document service infrastructure and collaboration RIA, which allows collaborators to declare and participate in document collaborations in an interactive, intuitive and dynamic way.
\end{abstract}

Keywords: service mashups, open document collaboration.

\section{Introduction}

Service mashups offer end-user-oriented compositions of APIs, content and data sources from the Web. However, early approaches that built on simplified programming languages (scripting) or software composition techniques (pipes, workflows) saw limited commercial success. To tap their full potential, service mashups need to become more intuitive, beneficial and easy-to-use for end-users. This requires higherlevel models closer to end-user applications. In this work we present a novel mashup approach called MoSaiC that is based on a document metaphor and tailored to support open collaboration of end-users. Document mashups do not only provide an intuitive means to utilize service composition techniques but also enable so far missing support for human-centric situational ad-hoc processes. The demo illustrates the utilization of the MoSaiC Web application, execution platform and integration infrastructure.

\section{Concept, Implementation and Benefits of MoSaiC}

MoSaiC supports coordinators to decompose collaborative tasks into contributions to be provided by collaborators or robots as Web-based services. MoSaiC mashups define the aggregation and orchestration of contributions into a joint document [1]. To this end, contribution services are mapped to formatted elements of the document 
structure. The coordination of different collaborators (e.g. causal or temporal relations of their contributions) is declared by ECA-based rules associating incoming and outgoing service messages.

Mashups are one-way document instances that are evolved by collaborating individuals in terms of structure, behavior and contents. This flexible model fits a variety of application scenarios including financial reporting, process documentation, product specification, research publication, education material/exercises and many more.

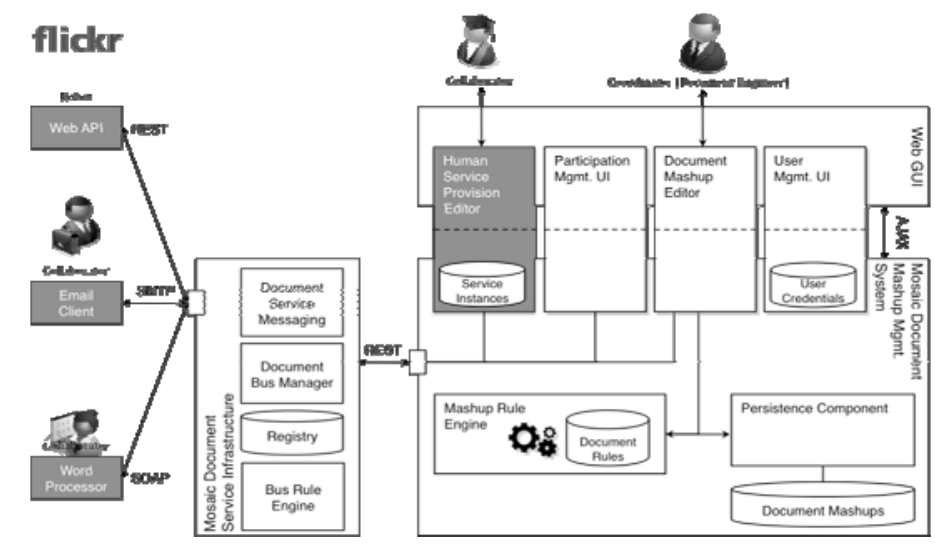

Fig. 1. Document Mashup System Architecture Overview (see screencast ${ }^{1}$ for UI pictures)

Figure 1 shows the MoSaiC architecture consisting of a) the mashup management system providing a rule-driven mashup engine and a Web GUI to initiate, maintain and participate in open document collaborations, b) the document service infrastructure enabling interaction (asynchronous messaging, dynamic routing) as well as management (service registry) and integration (adapters) of various Web-resources.

The MoSaiC approach offers an application-specific mashup model that is more pragmatic and intuitive than simplified software composition models. It leverages the potential of service mashups for unique support of open document collaborations. Beyond the specific MoSaiC application, we anticipate that the document metaphor is a promising basis to be leveraged by a future class of mashup approaches.

\section{Demonstration Overview}

The demo will showcase the utilization of MoSaiC for the use case of supporting collaborative research resulting in a paper. It illustrates the use of many concepts and features of the tool and infrastructure like ad hoc integration of human-provided and Web-based services or usage of coordination rules. We provide a screencast online ${ }^{1}$.

\section{Reference}

1. Schuster, N., Zirpins, C., Tai, S., et al.: A Service-Oriented Approach to Document-Centric Situational Collaboration Processes. In: Proc. WETICE 2009, pp. 221-226. IEEE, Los Alamitos (2009)

\footnotetext{
${ }^{1}$ www.zirpins.de/downloads/MosaicScreenCast.m4v (subject to frequent updates)
} 